\title{
The Wave Energy Up Conversion of Plasma Wave in Inhomogeneous Ionosphereic Plasma
}

\author{
P. N. Deka ${ }^{1}$, S. J. Gogoi ${ }^{2 *}$ \\ ${ }^{1}$ Department of Mathematics, Dibrugarh University, Dibrugarh, Assam, India \\ ${ }^{2}$ Department of Physics, Tinsukia College, Tinsukia, Assam, India
}

Received 17 April 2019, accepted in final revised form 21 July 2019

\begin{abstract}
Different types of instabilities are observed in the thermodynamically nonequilibrium Earth's ionosphere. Effective energy exchange process among waves may takes place through nonlinear interaction modes because of availability of free energy. We consider gradients in density and magnetic field is present in the system which support drift wave turbulence. In this study we concern on the wave energy up conversion of electrostatic nonresonant lower hybrid wave through plasma maser instability in the mid-altitude ionospheric region. We have formulated the growth rate of lower hybrid wave by VlasovPoisson mathematical frame and estimated its value by observational data.
\end{abstract}

Keywords: Plasma maser effect; Lower hybrid wave; Drift wave; Density gradient; Magnetic field gradient.

() 2019 JSR Publications. ISSN: 2070-0237 (Print); 2070-0245 (Online). All rights reserved.

doi: http://dx.doi.org/10.3329/jsr.v11i3.40982 J. Sci. Res. 11 (3), 339-350 (2019)

\section{Introduction}

The Earth's ionosphere is a wall-less plasma laboratory has weak particle collision rate and low temperature. Through experimental observations it has been found the existence of both the linear and nonlinear properties in this atmospheric region [1]. In different altitudes of this region, naturally occurring instabilities are observed and their nonlinear effects are recorded. Perturbation develops in this open ionospheric plasma system for the entry of energy and momentum fluxes through particles and waves like solar wind. Gradients of several physical parameters like density, temperature and magnetic field are existing in this thermodynamically nonequilibrium system. In this ionospheric plasma region geomagnetic field is fluctuating with respect to altitudes for the interaction among solar wind, magnetospheric dynamo, interplanetary magnetic fields and the presence of other irregularities [2]. Due to the presence of a rich class of free energy sources in the earth's environment several macro- and micro-instabilities are observed in this open

Corresponding author: satyajyotitsk@gmail.com 
inhomogeneous plasma system and investigated at lower and mid altitudes and in the auroral zone [3-6].

In the mid-altitude F-region weakly collisional ionospheric plasma is produced mainly through photo-ionisation process and has peak plasma density [7,8]. From satellite observational data, gradient drift instability and temperature drift instability are observed during turbulent geomagnetic field conditions within $30^{\circ}$ to $60^{\circ}$ geomagnetic latitude ranges in the mid-altitude ionospheric plasma [9]. Kelly [5] observed in inhomogeneous ionospheric region at the mid altitude, different entities like gravity waves, shear effects, drift waves and lower hybrid waves are contributing in turbulence processes. In several studies investigations on instabilities in an inhomogeneous magnetised plasma system associated with density and temperature gradients from nonlinear wave-particle interaction approach in both open and laboratory plasma environment as well as in homogeneous and inhomogeneous magnetised plasma system are carried on [10-17]. Deka [18] investigated on the amplification of Langmuir wave in presence of ion acoustic turbulence in inhomogeneous plasma in presence of magnetic field gradients through plasma maser effect.

In this study, for the first time, we are investigating on probable amplification of lower hybrid wave in the presence of drift wave in ionospheric region at mid-altitude taking magnetic fields gradients and density gradients through plasma-maser instability and wish to estimate the growth rate by using observational data. Here on the basis of weak turbulence theory, plasma maser effect which was also known as induced Bremsstrahlung instability is a mode coupling effect and associated with the linear Landau damping [19]. In this effect electrons are accelerated by resonant mode drift wave turbulent field. The accelerated electrons transfer its energy to nonresonant lower hybrid wave through a modulated field non-linearly. Also due to wave-particle-wave interaction a high frequency dissipative nonlinear force is developed which acts as driving force to the growth of nonresonant mode. For this theoretical investigation we are using VlasovPoisson mathematical framework to evaluate fluctuating parts of distribution functions, modulated electric field, nonlinear force and growth rate expression through Fourier transform and integration along unperturbed orbit methods.

\section{Formulation of the Problem}

Consider a semi-infinite bounded inhomogeneous plasma which is confined by a magnetic field in the presence of low frequency drift wave. As superimposing perturbation field we consider a high frequency lower hybrid wave to the system. Let the plasma density decreases in the $\mathrm{x}$-direction is balanced by a magnetic field that increases with $\mathrm{x}$.

For this system, the electron distribution function is considered as [20]

$$
f_{j o}=f_{j o}\left(v_{\perp}^{2}, v_{x}\right)\left[1-\varepsilon^{\prime}\left(x+\frac{v_{y}}{\Omega_{j}}\right)\right]
$$

Here 


$$
\varepsilon^{\prime}=-\left.\frac{1}{f_{j o}} \frac{\partial f_{j o}}{\partial x}\right|_{x=x_{0}} \text { is density gradient. }
$$

Here " $\mathrm{j}$ " is used to specify plasma particle specification.

$$
\begin{aligned}
& \Omega_{j}=\frac{e_{j} B_{o}}{m_{j} c} \text { is cyclotron frequency. } \\
& \varepsilon^{\prime \prime}=-\frac{1}{B_{o}} \frac{d B_{o}}{d x} \text { is magnetic field gradient. }
\end{aligned}
$$

In this problem neglecting temperature variation and their anisotropy, the density and magnetic field with gradients are taken as-

$$
\begin{aligned}
& n_{j}=n_{j o}\left(1-\varepsilon^{\prime} x\right) \\
& \vec{B}(x)=\hat{z} B_{o}\left(1+\varepsilon^{\prime \prime} x\right)
\end{aligned}
$$

\section{Mathematical Analysis}

The interaction of Lower hybrid wave with drift wave is generated by the Vlasov-Poison system of equations which are -

$$
\begin{aligned}
& {\left[\frac{\partial}{\partial t}+\vec{v} \cdot \frac{\partial}{\partial \vec{r}}-\frac{e_{j}}{m_{j}}\left(\vec{E}+\frac{\vec{v} \times \vec{B}}{c}\right) \cdot \frac{\partial}{\partial \vec{v}}\right] \vec{F}(\vec{r}, \vec{v}, t)=0} \\
& \vec{\nabla} \cdot \vec{E}=-4 \pi n_{j} e_{j} \int f(\vec{r}, \vec{v}, t) \cdot d \vec{v}
\end{aligned}
$$

The unperturbed distribution function for electrons, the unperturbed electric field and the unperturbed magnetic fields are taken as-

$$
\begin{aligned}
& F_{o j}=f_{o j}+\varepsilon f_{o j}+\varepsilon^{2} f_{2 j} \\
& \vec{E}_{o l}=\varepsilon \vec{E}_{l} \\
& \vec{B}_{o l}=\vec{B}_{0}(x)=\hat{z} B_{0}\left(1+\varepsilon^{\prime \prime} x\right)
\end{aligned}
$$

Where $f_{o j}$ is space and time averaged part of the distribution function, $f_{1 j}$ and $f_{2 j}$ are fluctuating parts due to low frequency drift wave turbulence, $\varepsilon_{\text {is ordering of the low- }}$ frequency drift wave turbulence field, electric field and wave vector of drift turbulence are

$$
\vec{E}_{l}=\left(E_{l \perp}, 0, E_{l \|}\right) \text { and } \vec{k}=\left(k_{\perp}, 0, k_{\|}\right) \text {. }
$$

Now, equation (2) can be written as

$$
\left[\frac{\partial}{\partial t}+\vec{v} \cdot \frac{\partial}{\partial \vec{r}}-\varepsilon \frac{e_{j}}{m_{j}} \vec{E}_{l} \cdot \frac{\partial}{\partial \vec{v}}-\frac{e_{j}}{m_{j}}\left(\frac{\vec{v} \times \vec{B}}{c}\right) \cdot \frac{\partial}{\partial \vec{v}}\right]\left(f_{o j}+\varepsilon f_{1 j}+\varepsilon^{2} f_{2 j}\right)=0
$$

To the order of $\varepsilon$, we have - 


$$
\left[\frac{\partial}{\partial t}+\vec{v} \cdot \frac{\partial}{\partial \vec{r}}-\frac{e_{j}}{m_{j}}\left(\frac{\vec{v} \times \vec{B}}{c}\right) \cdot \frac{\partial}{\partial \vec{v}}\right] f_{1 j}=\frac{e_{j}}{m_{j}} \vec{E}_{l} \cdot \frac{\partial}{\partial \vec{v}} f_{o j}
$$

Since the system is inhomogeneous in the $\mathrm{x}$-direction, here we consider wave propagation only in the $y-z$ plan. Equations of motion are [20]

$$
\begin{aligned}
& \frac{d \vec{r}^{\prime}}{d t^{\prime}}=\vec{v}^{\prime} \\
& \frac{d \vec{v}^{\prime}}{d t}=-\frac{e_{j}}{m_{j} c} \vec{v}^{\prime} \times \vec{B}_{o}\left(1+\varepsilon^{\prime \prime} x^{\prime}\right) \\
& \vec{r}^{\prime}\left(t^{\prime}=t\right)=\vec{r} \text { and } \vec{v}^{\prime}\left(t^{\prime}=t\right)=\vec{v}
\end{aligned}
$$

From equations (7), the particle orbits, under the boundary conditions $x^{\prime}(o)=x, \vec{v}^{\prime}(\tau=o)=\vec{v}$ are $[18,20]$

$$
\begin{aligned}
& x^{\prime}-x=\frac{v_{y}}{\left(1+\varepsilon^{\prime \prime} x-\frac{\varepsilon^{\prime \prime} v_{y}}{\Omega}\right)} \sin \left\{\theta+\left(1+\varepsilon_{j}^{\prime \prime} x-\frac{\varepsilon^{\prime \prime} v_{y}}{\Omega}\right) \tau\right\}-\frac{\varepsilon^{\prime \prime} v_{y}}{2 \Omega}\left[\frac{\sin 2 \theta}{\Omega_{j}} \sin \Omega{ }_{j} \tau+\frac{\cos (2 \theta+2 \Omega j)}{2 \Omega_{j}}+\frac{2 \cos \Omega{ }_{j} \tau}{\Omega_{j}} \cos ^{2} \theta\right] \\
& -\frac{v_{\perp} \sin \theta}{\left(1+\varepsilon^{\prime \prime} x-\frac{\varepsilon^{\prime \prime} v_{y}}{\Omega}\right)}+\frac{\varepsilon^{\prime \prime} v_{\perp}^{2}}{2 \Omega_{j}^{2}}\left(\frac{\cos 2 \theta}{2}+2 \cos ^{2} \theta\right)
\end{aligned}
$$$$
y^{\prime}-y=-\frac{v_{\perp}}{\left(1+\varepsilon^{\prime \prime} x-\frac{\varepsilon^{\prime \prime} v_{y}}{\Omega_{e}}\right)} \cos \left\{\theta+\left(1+\varepsilon^{\prime \prime} x-\frac{\varepsilon^{\prime \prime} v_{y}}{\Omega_{e}}\right) \tau\right\}-\frac{\varepsilon^{\prime \prime} v_{\perp}}{2 \Omega_{e}}\left[\tau-\frac{\sin 2 \theta}{\Omega_{e}} \cos \Omega_{e} \tau+\frac{\sin \left(2 \theta+2 \Omega_{e} \tau\right)}{2 \Omega_{e}}-\right.
$$$$
\left.\frac{2 \sin \Omega_{e} \tau}{\Omega_{e}} \cos ^{2} \theta\right]+\frac{v_{\perp} \cos \theta}{\left(1+\varepsilon^{\prime \prime} x-\frac{\varepsilon^{\prime \prime} v_{y}}{\Omega_{e}}\right)}-\frac{\varepsilon^{\prime \prime} v_{\perp}^{2}}{4 \Omega_{e}^{2}} \sin 2 \theta
$$$$
z^{\prime}-z=v_{\|} \tau
$$

Using Fourier transform and the method of characteristics [20], from equation (6) we have

$$
f_{\text {le }}(\vec{k}, \omega)=i \sum \frac{e}{m}\left[E_{l \|} \frac{\partial f_{o e}}{\partial v_{\|}} \mathbb{R}_{s, t}-\frac{m}{k_{\perp} T_{e}} E_{l \perp}\left\{1+\left(k_{\|\|} v_{\|}-\omega-\frac{\varepsilon^{\prime} k_{\perp} T_{e}}{m \Omega_{e}}\right) \mathbb{R}_{s, t}\right\} f_{o e}\right]
$$

where 


$$
\mathbb{R}_{s, t}=\sum_{s, t} \frac{J_{s}\left(\frac{v_{\perp} k_{\perp}}{1+\varepsilon^{\prime \prime} x-\frac{\varepsilon^{\prime \prime} v_{y}}{\Omega_{j}}}\right) J_{t}\left(\frac{v_{\perp} k_{\perp}}{1+\varepsilon^{\prime \prime} x-\frac{\varepsilon^{\prime \prime} v_{y}}{\Omega_{j}}}\right) \mathrm{e}^{[-i(s+t) \theta]}}{s\left(\frac{v_{\perp} k_{\perp}}{1+\varepsilon^{\prime \prime} x-\frac{\varepsilon^{\prime \prime} v_{y}}{\Omega_{j}}}\right)+\left(k_{\|} v_{\|}-\omega\right)+i 0^{+}}
$$

Let the quasi steady state is perturbed by the test nonresonant lower hybrid wave field $\mu \delta \vec{E}_{h}$ propagation vector $K=\left(0,0, K_{\|}\right)$, electric field $\delta E=\left(0,0, \delta E_{h}\right)$ and a frequency $\Omega$. Here electrostatic lower hybrid waves are directed perpendicular to magnetic fields $[21,22]$. Due to this perturbation total perturbed electric field, magnetic field and electric distribution function are

$$
\begin{aligned}
& \delta \vec{E}=\mu \delta \vec{E}_{h}+\mu \varepsilon \delta \vec{E}_{l h}+\mu \varepsilon^{2} \Delta \vec{E} \\
& \delta \vec{B}=0 \\
& \delta f_{j}=\mu \delta f_{h}+\mu \varepsilon \delta f_{l h}+\mu \varepsilon^{2} \Delta f
\end{aligned}
$$

Where $\delta E_{l h}$ and $\Delta \vec{E}$ are modulating fields, $\delta f_{h}$ is the fluctuating part due to high frequency lower hybrid wave, $\delta f_{l h}$ and $\Delta f$ are particle distribution function corresponds to modulating fields

Let, the operator

$$
\hat{L}=\frac{\partial}{\partial t}+\vec{v} \cdot \frac{\partial}{\partial \vec{r}}-\frac{e_{j}}{m_{j}}\left(\frac{\vec{v} \times \vec{B}_{o}}{c}\right) \cdot \frac{\partial}{\partial \vec{v}}
$$

Using equation (10) in Vlasov equation (2) for the perturbed state, we get -

$$
\begin{aligned}
& {\left[\hat{L}-\frac{e_{j}}{m_{j}}\left(\mu \vec{E}_{l}+\mu \delta \vec{E}_{h}+\mu \varepsilon \delta \vec{E}_{l h}+\mu \varepsilon^{2} \Delta \vec{E}\right) \cdot \frac{\partial}{\partial \vec{v}}\right] \times} \\
& \left(f_{o j}+\varepsilon f_{1 j}+\varepsilon^{2} f_{2 j}+\mu \delta f_{h}+\mu \varepsilon \delta f_{l h}+\mu \varepsilon^{2} \Delta f\right)=0
\end{aligned}
$$

To the order of $\mu$, we have-

$$
\hat{L} \delta f_{h}=\frac{e_{j}}{m_{j}} \delta \vec{E}_{h} \cdot \frac{\partial}{\partial \vec{v}} f_{o j}
$$

To the order of $\mu \varepsilon$, we have -

$$
\hat{L} \delta f_{l h}=\frac{e_{j}}{m_{j}} \vec{E}_{l} \cdot \frac{\partial}{\partial \vec{v}} \delta f_{h}+\frac{e_{j}}{m_{j}} \delta \vec{E}_{h} \cdot \frac{\partial}{\partial \vec{v}} \delta f_{1 j}+\frac{e_{j}}{m_{j}} \delta \vec{E}_{l h} \cdot \frac{\partial}{\partial \vec{v}} \delta f_{o j}
$$


To the order of $\mu \varepsilon^{2}$ and applying random phase approximation to omit second order quantities, we have -

$$
\hat{L} \Delta f=\frac{e_{j}}{m_{j}}\left[\vec{E}_{l} \cdot \frac{\partial}{\partial \vec{v}} \delta f_{l h}+\delta \vec{E}_{l h} \cdot \frac{\partial}{\partial \vec{v}} \delta f_{1 j}\right]
$$

Applying Fourier transform and integrating along the unperturbed orbit, we can evaluate the fluctuating part $\delta f_{h}$ of the distribution function due to high frequency lower hybrid wave $K=\left(0,0, K_{\|}\right)$over the particle trajectories. Here

$$
\delta f_{h}(\vec{K}, \Omega)=\frac{e_{j}}{m_{j}} \int_{-\alpha}^{0}\left(\delta E_{h} \frac{\partial f_{o j}}{\partial v_{\|}}\right) \mathrm{e}^{\left[i\left\{K_{1}\left(z^{\prime}-z\right)-\Omega \tau\right\}\right] d \tau}
$$

Using

$$
\frac{\partial f_{o j}}{\partial \vec{v}}=\left[-\frac{m_{j} \vec{v}}{T_{j}}-\hat{X} \frac{\varepsilon^{\prime}}{\Omega_{j}}\right]
$$

and for weak gradient

$$
\frac{\varepsilon^{\prime \prime} K_{\perp} T_{j}}{2 \Omega_{j} m_{j}} \ll 1
$$

We get after lengthy calculations-

$$
\delta f_{h}(\vec{K}, \Omega)=-i \sum \frac{e_{j}}{m_{j}} \delta E_{h} \frac{\frac{\partial f_{o j}}{\partial v_{\|}}}{\left(K_{\|} v_{\|}-\Omega\right)}
$$

Using Fourier transform and the method of characteristics to equation (14) we get-

$$
\begin{aligned}
& \delta f_{l h}(\vec{K}-\vec{k}, \Omega-\omega)=\sum \frac{e_{j}}{m_{j}} \int_{-\alpha}^{0}\left(\delta \vec{E}_{l h} \cdot \frac{\partial}{\partial \vec{v}} f_{o j}+\delta \vec{E}_{h} \cdot \frac{\partial}{\partial \vec{v}} f_{1 j}+\vec{E}_{l} \cdot \frac{\partial}{\partial \vec{v}} \delta f\right) \cdot \mathrm{e}^{\left[i\left((\vec{K}-\vec{k}) \cdot\left(\vec{r}^{\prime}-\vec{r}\right)-(\Omega-\omega) \tau\right]\right] d \tau} \\
& =I_{l h}^{1}+I_{l h}^{2}+I_{l h}^{3}
\end{aligned}
$$

Here after lengthy calculations we get

$$
\begin{gathered}
I_{l h}^{1}=\sum \frac{e_{j}}{m_{j}} \int_{-\alpha}^{0} \delta \vec{E}_{l h} \cdot \frac{\partial}{\partial \vec{v}} f_{o j} \cdot \mathrm{e}^{\left[i\left\{(\vec{K}-\vec{k}) \cdot\left(\vec{r}^{\prime}-\vec{r}\right)-(\Omega-\omega) \tau\right\}\right] d \tau} \\
=i \sum \frac{e_{j}}{m_{j}} \frac{\delta E_{l h}}{k_{\perp}} \frac{m_{j}}{T_{j}}\left[1+\left\{(\Omega-\omega)-\left(K_{\|}-k_{\|}\right) v_{\|}-\frac{\varepsilon^{\prime} T_{j} k_{\perp}}{\Omega_{j} m_{j}}\right\} \mathbb{Z}_{a, b}\right] f_{o j} \\
I_{l h}^{2}=\sum \frac{e_{j}}{m_{j}} \int_{-\alpha}^{0} \delta \vec{E}_{h} \cdot \frac{\partial}{\partial \vec{v}} f_{1 j} \cdot \mathrm{e}^{\left[i\left\{(\vec{K}-\vec{k}) \cdot\left(\vec{r}^{\prime}-\vec{r}\right)-(\Omega-\omega) \tau\right\}\right] d \tau} \\
=i \sum\left(\frac{e_{j}}{m_{j}}\right)^{2} \delta E_{h} \frac{\partial}{\partial v_{\|}}\left[E_{l \|} \frac{\partial f_{o j}}{\partial v_{\|}} \mathbb{R}_{s, t}-\frac{m_{j}}{k_{\perp} T_{j}} E_{l \perp}\left\{1+\left(k_{\|} v_{\|}-\omega-\frac{\varepsilon^{\prime} k_{\perp} T_{j}}{m_{j} \Omega_{j}}\right) \mathbb{R}_{s, t}\right\} f_{o j}\right] \mathbb{Z}_{a, b}
\end{gathered}
$$




$$
\begin{aligned}
& I_{l h}^{3}=\sum \frac{e_{j}}{m_{j}} \int_{-\alpha}^{0} \vec{E}_{l} \cdot \frac{\partial}{\partial \vec{v}} \delta f_{h} \mathrm{e}^{\left[i\left\{(\vec{K}-\vec{k}) \cdot\left(\vec{r}^{\prime}-\vec{r}\right)-(\Omega-\omega) \tau\right\}\right] d \tau} \\
& =-\sum\left(\frac{e_{j}}{m_{j}}\right)^{2} E_{l \perp} \delta E_{h} \frac{\frac{\partial f_{o j}}{\partial v_{\|}}}{\left(K_{\|} v_{\|}-\Omega\right)} \frac{m_{j}}{k_{\perp} T_{j}}\left[1+\left\{(\Omega-\omega)-\left(K_{\|}-k_{\|}\right) v_{\|}-\frac{\varepsilon^{\prime} T_{j} k_{\perp}}{m_{j} \Omega_{j}}\right\} \mathbb{Z}_{a, b}\right]- \\
& \sum\left(\frac{e_{j}}{m_{j}}\right)^{2} E_{l \|} \delta E_{h} \frac{\partial}{\partial v_{\|}}\left(\frac{\frac{\partial f_{o j}}{\partial v_{\|}}}{\left(K_{\|} v_{\|}-\Omega\right)}\right) \mathbb{Z}_{a, b}
\end{aligned}
$$

Where

$$
\mathbb{Z}_{a, b}=\sum_{a, b} \frac{J_{a}\left(\frac{v_{\perp} k_{\perp}}{1+\varepsilon^{\prime \prime} x-\frac{\varepsilon^{\prime \prime} v_{y}}{\Omega_{j}}}\right) J_{b}\left(\frac{v_{\perp} k_{\perp}}{1+\varepsilon^{\prime \prime} x-\frac{\varepsilon^{\prime \prime} v_{y}}{\Omega_{j}}}\right) \mathrm{e}^{[-i(a+b) \theta]}}{a\left(1+\varepsilon^{\prime \prime} x-\frac{\varepsilon^{\prime \prime} v_{y}}{\Omega_{j}}\right)+\left(K_{\|}-k_{\|}\right) v_{\|}-(\Omega-\omega)}
$$

By using Poisson's equation

$$
\vec{\nabla} \cdot \delta \vec{E}_{l h}=-4 \pi \sum e_{j} n_{j} \int \delta f_{l h} \cdot d \vec{v}
$$

the expression of modulated electric field $\delta \vec{E}_{l h}(\vec{K}-\vec{k})$ is

$$
\delta E_{l h}=-\sum \frac{4 \pi e_{j} n_{j}}{i R|\vec{K}-\vec{k}|}\left(\frac{e_{j}}{m_{j}}\right)^{2} \delta E_{h}\left\{\Delta^{1}+\Delta^{2}+\Delta^{3}\right\}
$$

Where

$$
\begin{aligned}
& \Delta^{1}=\int\left[-E_{l \perp} \frac{\frac{\partial f_{o j}}{\partial v_{\|}}}{\left(K_{\|\|} v_{\|}-\Omega\right)} \frac{m_{j}}{k_{\perp} T_{j}}\left[1+\left\{(\Omega-\omega)-\left(K_{\|}-k_{\|}\right) v_{\|}-\frac{\varepsilon^{\prime} T_{j} k_{\perp}}{m_{j} \Omega_{j}}\right\} \mathbb{Z}_{a, b}\right]\right] d v \\
& \Delta^{2}=\int\left[-E_{l \|} \frac{\partial}{\partial v_{\|}}\left(\frac{\frac{\partial f_{o j}}{\partial v_{\|}}}{K_{\|\|_{\|}}-\Omega}\right) \mathbb{Z}_{a, b}\right] d v \\
& \Delta^{3}=\int\left[\frac{\partial}{\partial v_{\|}}\left[E_{l \|} \frac{\partial f_{o j}}{\partial v_{\|}} \mathbb{R}_{s, t}-\frac{m_{j}}{k_{\perp} T_{j}} E_{l \perp}\left\{1+\left(k_{\|} v_{\|}-\omega-\frac{\varepsilon^{\prime} k_{\perp} T_{j}}{m_{j} \Omega_{j}}\right) \mathbb{R}_{s, t}\right\} f_{o j}\right] \mathbb{Z}_{a, b}\right] d v
\end{aligned}
$$


and

$$
R=1+\left(\frac{m_{j}}{T_{j}}\right) \frac{4 \pi n_{j} e_{j}^{2}}{m_{j}|\vec{K}-\vec{k}|} \int\left[1+\left\{(\Omega-\omega)-\left(K_{\|}-k_{\|}\right) v_{\|}-\frac{\varepsilon^{\prime} k_{\perp} T_{j}}{m_{j} \Omega_{j}}\right\} \mathbb{Z}_{a, b}\right] f_{o j} d v
$$

For the nonlinear interaction between low frequency resonant drift wave $\vec{k}=\left(k_{\perp}, 0, k_{\|}\right)$ and high frequency nonresonant lower hybrid wave $\vec{K}=\left(0,0, K_{\|}\right)$present in the system by means of weak turbulence theory a nonlinear force develop due to acceleration of electrons in the modulated electric field $\delta \vec{E}_{l h}$ and contribute in wave energy up conversion process [23].

From equation (14)-

$$
\hat{L} \Delta f=\frac{e_{j}}{m_{j}}\left[\vec{E}_{l} \cdot \frac{\partial}{\partial \vec{v}} \delta f_{l h}+\delta \vec{E}_{l h} \cdot \frac{\partial}{\partial \vec{v}} f_{1 j}\right]=\vec{F} \text { (Say) }
$$

Using Fourier transform and integrating along unperturbed orbits, we get -

$$
\begin{aligned}
& \Delta f=\int_{-\alpha}^{0} \vec{F}(\vec{K}, \Omega) \mathrm{e}^{\left\{i\left\{\vec{K} \cdot\left(\vec{r}^{\prime}-\vec{r}\right)-\Omega \tau\right\}\right] d \tau} \\
& =-i \frac{F}{K_{\|}\left(z^{\prime}-z\right)-\Omega}
\end{aligned}
$$

The nonlinear force $F_{N h}$ acting on unit volume of particles can be written as

$$
\begin{aligned}
\vec{F}_{N h} & =m_{j} n_{j o} \Omega \int \frac{F}{K_{\|} v_{\|}-\Omega} \vec{v} d \vec{v} \\
& =m_{j} n_{j 0} \Omega \int\left\langle\frac{e_{j}}{m_{j}}\left[\vec{E}_{l} \cdot \frac{\partial}{\partial \vec{v}} \delta f_{l h}+\delta \vec{E}_{l h} \cdot \frac{\partial}{\partial \vec{v}} \delta f_{1 j}\right]\right) \times \frac{1}{K_{\|} v_{\|}-\Omega} \vec{v} d \vec{v}
\end{aligned}
$$

where $\langle\ldots \ldots \ldots . . .$.$\rangle represents ensemble average.$

The Z-component of this nonlinear force is-

$\vec{F}_{N h z}(\vec{K}, \Omega)=\vec{F}_{N h z 1}(\vec{K}, \Omega)+\vec{F}_{N h z 2}(\vec{K}, \Omega)$

where

$$
\begin{aligned}
& F_{N h z 1}=e_{j} n_{j 0} \Omega \int\left\langle\vec{E}_{l} \cdot \frac{\partial}{\partial \vec{v}} \delta f_{l h}\right\rangle \frac{1}{K_{\|} v_{\|}-\Omega} v_{\|} d v \\
& F_{N h z 2}=e_{j} n_{j 0} \Omega \int\left\langle\delta \vec{E}_{l h} \cdot \frac{\partial}{\partial \vec{v}} \delta f_{1 j}\right\rangle \frac{1}{K_{\|} v_{\|}-\Omega} v_{\|} d v
\end{aligned}
$$

Here, the direct coupling term contributes to the nonlinear component force $F_{N h z 1}$ and the other high frequency nonlinear component force $F_{N h z 2}$ comes from contributions of polarization coupling term. From earlier investigations $[18,24]$ it is found that for the 
growth of plasma wave in the presence of lower hybrid wave in inhomogeneous magnetised plasma the polarization coupling term dominates over the direct coupling term. In our present discussion we retain only the nonlinear force component $F_{N h z 2}(\vec{K}, \Omega)$ only.

To evaluate $F_{N h z 2}(\vec{K}, \Omega)$ we consider the plasma maser effect arises from resonant electrons under the condition $\omega=\vec{k} \cdot \vec{v}$ between lower hybrid wave and drift wave turbulence. It is also assumed that (i) $\Omega<K_{\|} v_{\|}$(ii) the gyro angle $\theta$ should be very small and (iii) keeping $\mathrm{a}=\mathrm{b}=\mathrm{s}=\mathrm{t}=0$ to retaining the most dominant Bessel's terms. Here we have used the relation

$$
\operatorname{Im} \frac{1}{k v-\omega+i o^{+}}=-\pi i \delta(\omega-k v)
$$

and

$$
\int_{-\infty}^{\infty} G(v) \delta(\omega-k v)=\left(\frac{1}{|k|} G\left(\frac{\omega}{k}\right)\right)
$$

Here $J_{0}\left(\frac{v_{\perp} K_{\perp}}{1+\varepsilon^{\prime \prime} x-\frac{\varepsilon^{\prime \prime} v_{y}}{\Omega_{j}}}\right)=1-\frac{\left(\frac{v_{\perp} K_{\perp}}{1+\varepsilon^{\prime \prime} x-\frac{\varepsilon^{\prime \prime} v_{y}}{\Omega_{j}}}\right)^{2}}{4}$

From observational data it is found that magnetic gradients in the ionospheric region is very much low, we can neglect $\varepsilon^{\prime \prime}=0$.

After lengthy calculations and keeping dominant terms only, from equation (21) we get approximate expression

$$
\delta E_{l h}=\left(\frac{e_{j}}{m_{j}}\right) E_{l \perp} \delta E_{h} \frac{K_{\|}\left(K_{\|}-k_{\|}\right)^{2}}{k_{\|}^{2}} \frac{\omega_{p j}^{2}}{\Omega_{j}^{3}\left(\Omega_{j}-\Omega\right)}
$$

where at the lowest order approximation we obtain

$$
\frac{1}{\Re|\vec{K}-\vec{k}|^{2}} \simeq \frac{1}{k_{\|}^{2}}
$$

Using equations (9) and (28) and to consider only polarization coupling term we get from equation (25)

$$
\begin{aligned}
& \vec{F}_{N h z}(\vec{K}, \Omega)=\vec{F}_{N h z 2}(\vec{K}, \Omega) \\
& =i e_{j} n_{j o} \delta E_{h}\left(\frac{e_{j}}{m_{j}}\right)^{2} \frac{K_{\|}\left(K_{\|}-k_{\|}\right)^{2}}{k_{\|}^{2}} \frac{\omega_{p j}^{2}}{\Omega_{j}^{3}\left(\Omega_{j}-\Omega\right)} E_{l \|}\left(E_{l \perp} \frac{\varepsilon^{\prime}}{\Omega_{j}}+2 E_{l \|} \frac{v_{d}}{v_{j}^{2}}\right) \mathrm{e}^{\left\{-\left(\frac{v_{d}}{v_{j}}\right)^{2}\right\}}
\end{aligned}
$$

In the presence of nonlinear force term, by using the method of Chen [25], the momentum equation of electron as 


$$
m_{j} n_{j}\left(\frac{\partial \vec{v}_{j}}{\partial t}+\vec{v}_{j} \cdot \vec{\nabla} v_{j}\right)=-e_{j} n_{j} \vec{\nabla} \phi+\vec{F}_{N h}
$$

where $v_{j}, n_{j}$ and $\phi$ are the density, velocity and potential of charge particles.

Equation (31) is linearized on the steady state condition $n_{j}=n_{j o}$ and $v_{j}=v_{j o}=0$, and applying Fourier transformations, the first order z-component of momentum equation becomes

$$
-i m_{j} n_{j o} \Omega v_{j 1 z}=-i e_{j} n_{j o} \chi \phi_{1}+F_{N h z}
$$

From Boltzmann relation, first order charge particle density fluctuation is

$$
\delta n_{j}=n_{j o} \frac{e_{j} \phi_{1}}{k_{B} T_{j}}
$$

The continuity equation for electron is

$$
\frac{\partial n_{j}}{\partial t}=-\vec{\nabla} \cdot\left(n_{j} v_{j}\right)
$$

After linearizing equation (39) become

$$
v_{j z}=-\frac{\Omega \delta n_{j}}{n_{j o} \chi}
$$

Using equation (37),(38) and (40), we get

$$
\Omega^{2}=\chi^{2}\left(-\frac{k_{B} T_{j}}{m_{j}}\right)+i \frac{\chi}{m_{j}} \frac{F_{N h z}}{\delta n_{j}}
$$

is the dispersion relation of electrostatic lower hybrid wave in the presence of drift wave turbulence.

As neglecting nonlinear frequency shift, from equation (36) we get real frequency of lower hybrid wave

$$
\Omega_{r}=\chi\left(-\frac{k_{B} T_{j}}{m_{j}}\right)^{\frac{1}{2}}
$$

and growth rate of lower hybrid wave

$$
\gamma=\frac{\chi}{2 m_{j} \Omega_{r}} \frac{F_{N h z}}{\delta n_{j}}
$$

Now using equations (30), (33) and (37) in equation (38) we obtain

$$
\frac{\gamma}{\Omega}=\frac{1}{2}\left(\frac{e_{j}}{m_{j}}\right)^{2}\left(\frac{m_{j}}{k_{B} T_{j}}\right)^{\frac{1}{2}} \Omega \frac{\omega_{p j}^{2}}{\Omega_{j}^{3}} \frac{K_{\|}\left(K_{\|}-k_{\|}\right)^{2}}{k_{\|}^{2}} E_{l \|}\left(E_{l \perp} \frac{\varepsilon^{\prime}}{\Omega_{j}}+2 E_{l \|} \frac{v_{d}}{v_{j}^{2}}\right) \mathrm{e}^{\left\{-\left(\frac{v_{d}}{v_{j}}\right)^{2}\right\}}
$$

Equation (39) estimates the growth rate of lower hybrid wave with drift wave turbulence from polarization coupling term in inhomogeneous plasma.

From observation it is found that $\left|E_{l \perp}\right| \gg\left|E_{l \|}\right|$ for drift wave turbulence, for this neglecting $E_{l \|}$ factor, equation (39) becomes 


$$
\frac{\gamma}{\Omega}=\frac{1}{2}\left(\frac{e_{j}}{m_{j}}\right)^{2}\left(\frac{m_{j}}{k_{B} T_{j}}\right)^{\frac{1}{2}} \Omega \frac{\omega_{p j}^{2}}{\Omega_{j}^{3}} \frac{K_{\|}\left(K_{\|}-k_{\|}\right)^{2}}{k_{\|}^{2}} E_{l \|} E_{l \perp} \frac{\varepsilon^{\prime}}{\Omega_{j}} \mathrm{e}^{\left\{\left(-\frac{v_{d}}{v_{j}}\right)^{2}\right\}}
$$

For the weak density plasma region we can put $\varepsilon^{\prime}=0$ and equation (39) becomes

$$
\frac{\gamma}{\Omega}=\left(\frac{e_{j}}{m_{j}}\right)^{2}\left(\frac{m_{j}}{k_{B} T_{j}}\right)^{\frac{1}{2}} \Omega \frac{\omega_{p j}^{2}}{\Omega_{j}^{3}} \frac{K_{\|}\left(K_{\|}-k_{\|}\right)^{2}}{k_{\|}^{2}} E_{l \|}{ }^{2} \frac{v_{d}}{v_{j}^{2}} \mathrm{e}^{\left\{\left(-\frac{v_{d}}{v_{j}}\right)^{2}\right\}}
$$

\section{Results and Discussion}

In this study by using Vlasov-Poisson system of equations, the dispersion relation of electrostatic lower hybrid wave in the presence of drift wave turbulence is derived in inhomogeneous magnetised plasma. We have considered polarization coupling term of nonlinear dispersion for estimation of growth rate of lower hybrid wave. The issue of plasma instabilities in ionospheric region at mid-altitude in the presence of drift wave turbulence is relevant at present. To obtain an estimation of the growth rate of lower hybrid wave, we have considered the following observational data:

1) The Plasma parameters and Lower hybrid wave parameters in space [26,27]:

$$
T_{e}=400 \mathrm{~K}, \Omega=\Omega_{e}=10^{6} \mathrm{~s}^{-1}, \frac{\omega_{p e}}{\Omega_{e}} \approx 0.3, K_{\|}=10^{-3} \mathrm{~m}^{-1}
$$

2) The Drift wave Parameters in space [28]:

$$
k_{\|} 10^{-3} \mathrm{~m}^{-1}, k_{\perp}=10^{-5} \mathrm{~m}^{-1}, v_{d}=10^{6} \mathrm{~ms}^{-1}
$$

3) The observational electrostatic plasma wave dc electric field intensity in the mid altitude ionospheric region [29]:

$$
k_{\|}=10^{-2} V m^{-1}, E_{l \perp}=10^{-1} V m^{-1}
$$

Considering density gradient $\varepsilon^{\prime}=0.1$ the growth rate for lower hybrid wave in presence of drift wave turbulence in inhomogeneous ionospheric plasma from equation (40) as

$$
\begin{aligned}
& \frac{\gamma}{\Omega} \approx 10^{-5} \text {. } \\
& \text { as } K_{\|} \gg K_{\|}-k_{\|} \text {is assumed to take } K_{\|}-k_{\|} \approx 10^{-5} .
\end{aligned}
$$

\section{Conclusion}

In this theoretical investigating work it is found that drifting particles takes part through nonlinear energy exchange mode in the wave energy amplification process of high frequency plasma wave in the presence of both density and magnetic field strength gradients. After considering weak magnetic field gradients $(\approx 0)$ in the inhomogeneous ionospheric zone the wave energy upconversion process of nonresonant wave may also be effective in the presence of density gradients. 


\section{References}

1. P. Stubbe and T. Hagfors, Survey in Geophys. 18, 57 (1997). https://doi.org/10.1023/A:1006583101811

2. S. Bhardwaj, P. A. Khan, R. Atulkar, and P. K. Purohit , J. Sci. Res. 10, 133 (2018). https://doi.org/10.3329/jsr.v10i2.34509

3. D. T. Farley, Ann. Geophys. 27, 1509 (2009). https://doi.org/10.5194/angeo-27-1509-2009

4. B. G. Fejer and M. C. Kelley, Rev. Geophys. 18, 401 (1980). https://doi.org/10.1029/RG018i002p00401

5. M. C. Kelley, The Earths Ionosphere: Plasma Physics and Electrodynamics (Academic Press Inc: Harcourt Brace Jovanovich Publishers, 2009).

6. R. A. Truemann and W. Baumjohann, Advanced Space Plasma Physics, Revised Edition, (Imperial College Press, London, 2001).

7. V. G. Istomin, Ann. Geophys. 22, 255 (1966). https://doi.org/10.1002/1097-4679(196604)22:2<255::AID-JCLP2270220245>3.0.CO;2-N

8. C. A. Reber and M. Nicolet, Planet. Space Sci. 13, 617 (1965). https://doi.org/10.1016/0032-0633(65)90043-7

9. S. Basu, S. Basu, J. J. Makela, E. MacKenzie, P. Doherty, J. W. Wright, F. Rich, M. J. Keskinen, R. E. Sheehan, and A. J. Coster, J. Geophys. Res. 113, A00A06 (2008).

10. E. V. Mishin, W. J. Burke, C. Y. Huang, and F. J. Rich, J. Geophys. Res. 108(AB), 1309 (2003). https://doi.org/10.1029/2002JA009793

11. M. J. Keskinen and S. Basu, Geophys. Res. Lett. 31, L09811 (2004). https://doi.org/10.1029/2003GL019368

12. A. Eltrass and W. A. Scales, J. Geophys. Res. Space Phys. 119, 78897901 (2014). https://doi.org/10.1002/2014JA020314

13. S. A. Kaplan and V. N. Tsytovich, Plasma Astrophysics (Pergamon Press, New York, 1973).

14. I. Alexe-Proced. of Workshop on Recent Advances in Plasma Physics (Indian Academy of Sciences, Bangalore, India, 1977), pp.131.

15. P. N. Deka and A. Borgohain, J. Plasma Phys. 78, 515 (2012). https://doi.org/10.1017/S0022377812000207

16. M. Singh and P. N. Deka, Phys. Plasma 12, 102304 (2005). https://doi.org/10.1063/1.2087587

17. P. N. Deka and J. K. Deka, J. Plasma Energy 37, 6 (2018).

18. P. N. Deka, Ph. D. Thesis (Gauhati University, Assam. India, 1999).

19. L. D. Landau, J. Phys. 10, 25 (1946). https://doi.org/10.3406/ingeo.1946.5138

20. N. A. Krall and A. W. Trivelpiece, Principles of Plasma Physics (McGrawHill Book Company, USA, 1973).

21. T. M. Stix, The Theory of Plasma Waves (McGraw Hill, New York, 1962).

22. R. A. Treumann and W. Baumjohann, Advanced Space Plasma Physics (Imperial College Press, London, 2001).

23. M. Nambu, Laser Part. Beams, 1, 427 (1983). https://doi.org/10.1017/S0263034600000513

24. M. Singh and P. N. Deka, Pramana, 66, 547 (2006). https://doi.org/10.1007/BF02704498

25. F. Chen, Introduction to Plasma Physics, (Plenum, New York, 1974).

26. M. Malingre, R. Pottelette, N. Dobouloz, P. A. Lindqvist, G. Holmgren, and B. Apricio. Geophys. Res. Lett. 19, 1339 (1992). https://doi.org/10.1029/92GL01154

27. D. A. Gurnett and L. A. Frank, J. Geophys. Res. 82, 1031 (1977). https://doi.org/10.1029/JA082i007p01031

28. P. K. Shukla, G. T. Birk, and R. Bingham, Geophys. Res. Lett. 22, 671 (1995). https://doi.org/10.1029/95GL00360

29. V. M. Sorokin, A. K. Yaschenko, and M. Hayakawa, Natural Hazarards Earth System Sci. 5, 661 (2005). https://doi.org/10.5194/nhess-5-661-2005 